\title{
Tenapanor: new approach to counter irritable bowel syndrome with constipation
}

\author{
D. B. S. Brashier ${ }^{1}$, Ajay Kumar ${ }^{3}$, Anuj Singh ${ }^{2 *}$, Uday Pratap ${ }^{4}$
}

\author{
${ }^{1}$ Department of Pharmacology, ${ }^{2}$ Department of Anaesthesiology and Critical Care, Government Hospital, Joshimath, \\ Uttarakhand, India \\ ${ }^{3}$ Department of Anaesthesiology and Critical Care, Military Hospital, Dehradun, Uttarakhand, India \\ ${ }^{4}$ Department of Surgery, Command Hospital, Lucknow, Uttar Pradesh, India
}

Received: 26 April 2020

Accepted: 06 June 2020

\section{*Correspondence:}

Dr. Anuj Singh,

Email: anujsingh.doc@gmail.com

Copyright: (c) the author(s), publisher and licensee Medip Academy. This is an open-access article distributed under the terms of the Creative Commons Attribution Non-Commercial License, which permits unrestricted non-commercial use, distribution, and reproduction in any medium, provided the original work is properly cited.

\begin{abstract}
Irritable bowel syndrome (IBS) is a functional gastrointestinal disorder chronic in nature and characterized predominantly by abdominal pain or discomfort associated with altered bowel habits, diagnosis requires characteristic symptoms during the last 3 months and onset $\geq 6$ months ago. Symptom-based approaches for functional bloating, constipation and diarrhea are best utilised to identify IBS. IBS with constipation exerts significant impairment on work productivity by hampering quality of life. Inadequate relief by existing modalities, persistent hard stools and visceral abdominal pain demanded further clinical research. Tenanapor a novel molecule acts locally on gastrointestinal sodium/hydrogen exchanger isoform 3 (NHE3), an antiporter a counter transporter and exert antinociceptive effects on visceral sensation thereby decreases the frequency of abdominal pain. Action on NHE3 receptors located on small intestine and colon's apical surface reduces the absorption of sodium and phosphate, with minimal systemic exposure. NHE3 Inhibition induced sodium absorption results in increase in water secretion into intestinal lumen resultant an accelerated intestinal transit time and softer stool consistency. Most common adverse reactions $(\geq 2 \%)$ are diarrhea, abdominal distension, flatulence and dizziness. The drug is metabolised mainly by CYP3A4/5 and excreted in feaces (70\%) and urine (7\%). Tenapanor's minimal systemic absorption is likely to be associated with a relatively inert safety and tolerability profile. Based on positive results from the phase III T3MPO trial program, tenapanor demonstrated promising results for IBS-C management and received US Food and Drug Administration approval as IBSRELA @ Ardelyx Pharma in September 2019 and augment existing modalities for management of IBS-C.
\end{abstract}

Keywords: Tenapanor, Inflammatory bowel syndrome, NHE3 antagonist, Constipation

\section{INTRODUCTION}

Irritable bowel syndrome (IBS) is an idioathic gastrointestinal (GI) disorder chronic in nature and characterized predominantly by abdominal pain or discomfort associated with altered bowel habits. Currently followed guidelines for clinical diagnosis of IBS have been formulated by many organisations, as Rome's foundation, American Society of Gastroenterologists,
American Gastroenterological Association. IBS also called as functional bowel disease has been associated with clinical spectrum of abdominal pain or discomfort associated with altered bowel habits and duration for more than 3 months and onset at least $>6$ months prior (Figure 1). The classification is done into subtypes on basis of bristol stool forms. Alarming symptoms suggest the possibility of structural disease, but do not necessarily negate a diagnosis of a functional bowel disorder. IBS is 
best identified with symptom based approaches for functional bloating, functional constipation, and functional diarrhea. ${ }^{1}$ The prevalence of IBS globally is 7$21 \%$ and amongst these, one third of the cases are constipation-predominant IBS (IBS-C)..$^{2-4}$ According to stool consistency IBS is sub classified as: IBS with constipation (IBS-C); IBS with diarrhea (IBS-D); mixed IBS (IBS-M) and un subtyped IBS (IBS-U). IBS though not associated with mortality but it does greatly hamper quality of life. ${ }^{5}$

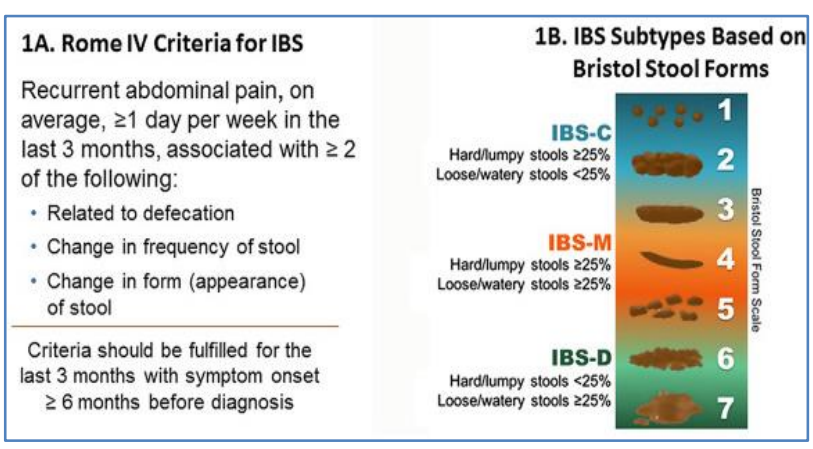

Figure 1: Rome foundation and Bristol stool forms criteria to diagnose IBS and sub types. ${ }^{4}$

There has been comparison of IBS-C with respect to negative impact and concluded impact being often similar or even worse than observed in chronic diseases as diabetes mellitus, rheumatoid arthritis. ${ }^{7-9}$ IBS-C diagnosis and formulation of treatment plan presents significant challenge due to the dynamic and diverse symptoms associated with clinical presentation. ${ }^{10}$ Historically management of IBS-C by laxatives, dietary fiber, and stool softeners lacked substantial evidences for their efficacy and low treatment satisfaction has been reported. ${ }^{10,11}$ The latest available modalities targeting IBS$\mathrm{C}$ symptoms include the guanylate cyclase-C receptor agonist linaclotide and the selective chloride channel activator lubiprostone, both drugs display least systemic absorption and target the GI tract. ${ }^{12-14}$ Inspite of achievement of primary end point of treatment for IBS-C, that is improvement in stool consistency and abdominal pain by lubiprostone and linaclotide, scope for therepeutic options still remains unachieved in patients with residual symptoms. ${ }^{12-14}$

Tenapanor is a novel, small-molecule inhibitor of the GI sodium/hydrogen exchanger isoform 3 (NHE3) and may exert antinociceptive effects on visceral sensation. ${ }^{15-17}$ Tenapanor acts on the receptors located on apical surface of the GI tract with resultant reduction in the absorption of sodium and phosphate, with minimal systemic drug exposure. ${ }^{15}$ Enhanced intestinal fluid volume and transit is demonstrated due increased sodium retention in gut, evident by softer stools and an increase in the frequency of bowel movements. The US Food and Drug Administration (FDA) has approved Ardelyx's IBSRELA (tenapanor) in September 2019 for the treatment of IBS-C.

\section{MECHANISM OF ACTION}

Tenapanor tablets contain tenapanor hydrochloride as an active ingredient. Tenapanor hydrochloride is a sodium/hydrogen exchanger 3 (NHE3) inhibitor for oral use. ${ }^{15-17}$ The chemical name for Tenapanor hydrochloride is 12,15-Dioxa-2,7,9-triazaheptadecanamide, 17-[3-[(4S)6,8dichloro-1,2,3,4-tetrahydro-2-methyl-4-

isoquinolinyl]phenyl]sulphonyl] amino]-N-[2-[2-[2[3[(4S)-6,8-dichloro-1,2,3,4-tetrahy-dro-2-methyl-

4isoquinolinyl]phenyl]sulphonyl]amino] ethoxy] ethoxy]ethoxy] ethyl]-8-oxo-, hydrochloride (1:2). ${ }^{15-17}$

Tenapanor hydrochloride has with molecular formula of C50H68Cl6N8010S2, the molecular weight of 1218 Daltons, and the chemical structure as depicted in (Figure 2). ${ }^{17}$

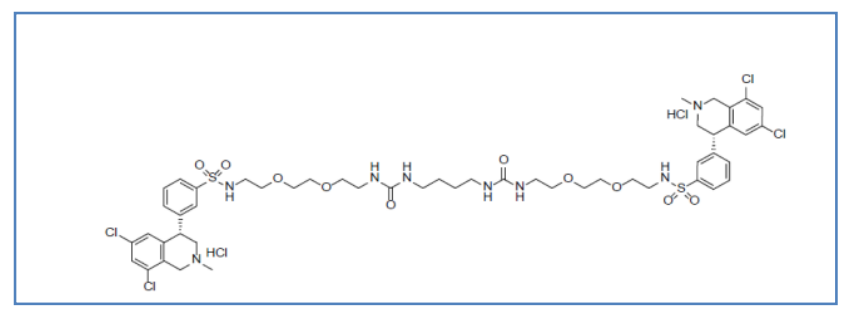

Figure 2: Chemical structure of Tenapanor.

The sodium/hydrogen exchanger (NHE) family of proteins facilitates the electro-neutral exchange of sodium ions for intracellular protons across membranes throughout the GI tract. ${ }^{18}$ Tenapanor is a locally acting inhibitor of the sodium/hydrogen exchanger 3 (NHE3), an antiporter, which is expressed on the apical surface of the small intestine and colon primarily responsible for the absorption of dietary sodium. ${ }^{15}$ Inhibition of NHE3 in the gut by tenapanor reduces absorption of gastrointestinal sodium, resulting in an increase in stool fluid content. ${ }^{15,18}$ The studies conducted in vitro and on animals indicated its major metabolite, M1, is inactive against NHE3. NHE3 receptor inhibition on the apical surface of the enterocytes due to tenapanor, reduces absorption of sodium from the small intestine and colon, consequently increases water secretion into the intestinal lumen, which accelerates intestinal transit time and results in a softer stool consistency. Tenapanor has been effective in reducing abdominal pain, by regulating visceral hypersensitivity. ${ }^{15}$

\section{CLINICAL PHARMACOLOGY}

Tenapanor twice daily oral dosage regimen is minimally absorbed. tenapanor and its major metabolite, M1, is bound to plasma protein, $99 \%$ and $97 \%$, respectively, in vitro. ${ }^{16}$ With high bioavailability following single or repeated oral dosage of tenapanor $50 \mathrm{mg}$ twice daily, Tenapanor plasma concentrations remained below the limit of quantitation (less than $0.5 \mathrm{ng} / \mathrm{ml}$ ) in the majority of samples from under evaluation subjects. ${ }^{17}$ 
Tenapanor is metabolized primarily by CYP3A4/5 and low levels of its major metabolite, M1, are detected in plasma. ${ }^{17}$ The Cmax of M1 is approximately $13 \mathrm{ng} / \mathrm{ml}$ after single dose of Tenapanor $50 \mathrm{mg}$ and $15 \mathrm{ng} / \mathrm{ml}$ at steady state following repeated dosing of Tenapanor 50 mg twice daily in subjects. ${ }^{17}$ Following administration of a single $15 \mathrm{mg}$ radiolabeled C-Tenapanor dose to healthy subjects, approximately $70 \%$ of the radioactivity was excreted in feces within 120 hours post-dose and $79 \%$ within 240 hours post-dose, mostly as the parent drug accounting for $65 \%$ of dose within 144 hours postdose. ${ }^{14,18}$ Approximately $9 \%$ of the administered dose was recovered in urine, primarily as metabolites. M1 is excreted in urine unchanged accounting for $1.5 \%$ of dose within 144 hours post-dose. ${ }^{17}$

Further to its pharmacodynamic effects Tenapanor's minimal systemic absorption is likely to be associated with a relatively inert safety and tolerability profile. ${ }^{19,20}$

Cross-study comparison, patients on hemodialysis (eGFR less than $15 \mathrm{ml} / \mathrm{min} / 1.73 \mathrm{~m}^{2}$ ) plasma concentrations of M1 in end-stage renal disease was not notably different from plasma concentrations of M1 in those of healthy subjects given comparable doses of tenapanor. ${ }^{19,20}$

\section{DRUG DOSAGE AND FORMULATION}

Tenapanor tablets contain $50 \mathrm{mg}$ of tenapanor (equivalent to $53.2 \mathrm{mg}$ of tenapanor hydrochloride). Inactive ingredients in the tablet includes colloidal silicon dioxide, hypromellose, low-substituted hydroxypropyl cellulose, microcrystalline cellulose, propyl gallate, stearic acid, tartaric acid powder, Titanium dioxide and Triacetin. ${ }^{20}$

The recommended dosage of tenapanor in adults is $50 \mathrm{mg}$ orally twice daily. ${ }^{20,21}$ Tenapanor has to be taken in adherence to meals regimen, immediately prior to breakfast or the first meal of the day and immediately prior to dinner, since the 24 hour $\mathrm{Na}+$ excretion in study subjects taking tenapanor 5-10 minutes prior to meal was higher than taking same dosage in fed condition. ${ }^{19,21}$ If a dose is missed, skip the missed dose and take the next dose at the regular time. Taking commulative dosage to compensate missed dose must not be taken in any scenario. ${ }^{19,21}$

\section{DRUG INTERACTION}

Tenapanor is metabolised primarily by CYP3A4/5 and and its active matabolite is M1 and excreted mainly in faeces predominantly and amller fraction in Urine. ${ }^{22}$ Studies Tenapanor and M1 did not inhibit CYP1A2, CYP2B6, CYP2C8, CYP2C9, CYP2C19, and CYP2D6 in vitro. Tenapanor and M1 did not induce CYP1A2 and CYP2B6 in vitro. ${ }^{22}$ CYP3A4 enzyme was unaffected and demonstrated no inhibition or induction using Midazolam as a substrate when Tenapanor $50 \mathrm{mg}$ was administered twice a day for 13 days in healthy subjects. ${ }^{22}$
Tenapanor $50 \mathrm{mg}$ single dose co administration with repeated doses of Itraconazole $200 \mathrm{mg}$, a CYP3A4 inhibitor, mean AUC and Cmax of M1 was decreased $50 \%$ in healthy subjects. And plasma concentrations of Tenapanor mostly remained below the limit of quantitation (less than $0.5 \mathrm{ng} / \mathrm{ml}){ }^{23}$

Tenapanor and M1 did not inhibit P-gp, BCRP, OATP1B1, and OATP1B3. M1 did not inhibit OAT1, OAT3, OCT2, MATE1, and MATE2-K. ${ }^{24}$ M1 is a substrate of P-gp. Tenapanor is not a substrate of $\mathrm{P}-\mathrm{gp}$, BCRP, OATP1B1, and OATP1B3 and M1 is not a substrate of BCRP, OAT1, OAT3, OCT2, MATE1 and MATE2-K. ${ }^{24}$ PepT1 activity had no significant change using cefadroxil as a substrate when Tenapanor $50 \mathrm{mg}$ was administered twice a day for 12 days in healthy subjects. $^{25}$

\section{SPECIAL CONSIDERATION AND SIDE EFFECTS}

\section{Pregnancy}

Tenapanor is least absorbed systemically on oral administration, and plasma concentrations remain below the limit of quantification (less than $0.5 \mathrm{ng} / \mathrm{ml}$ ). ${ }^{22}$ Maternal use henceforth is not expected to result in fetal exposure. Tenapanor exposure data obtained from a small number of pregnant women have documented drug associated risk for major birth defects, miscarriage, or adverse maternal or fetal outcomes. ${ }^{22}$

\section{Lactation}

Tenapanor secretion in either human or animal milk, its effects on milk production or its effects on the breastfed infant is still not being observed and there's paucity of data on said subject. In view of minimal systemic absorption, the drug will not yield clinically relevant exposure to breastfed infants. ${ }^{23}$

\section{Pediatric use}

Tenapanor is contraindicated in patients less than 6 years of age. ${ }^{25}$ Tenapanor is to be avoided in age group of patients, less than 6 years to 12 years. The safety and effectiveness of tenapanor in patients less than 18 years of age needs to be established and backed by clinical trials and evidences. ${ }^{25}$

\section{ADVERSE EFFECTS}

Tenapanor overdose may cause gastrointestinal adverse effects such as diarrhea as a result of exaggerated pharmacology and compounded risk for dehydration if diarrhea is severe or prolonged. ${ }^{26}$ The most common side effects involving more than $2 \%$ exposed population of tenapanor, include diarrhoea, abdominal distension, flatuelence and dizziness. ${ }^{26}$ In patients of chronic kidney disease, hyperkalemia was noted. Incidence rate of less 
than $2 \%$ mainly rectal bleeding and abnormal gastrointestinal sounds are not a rare possibility. ${ }^{20}$

\section{CONTRAINDICATION}

Tenapanor is contraindicated in patients of pediatric age group age less than 6 years, owing to higher risk of dehydartion and patients with known or suspected gastrointestinal obstruction. ${ }^{1}$

\section{CLINICAL TRIALS}

The safety data obtained and tabulated below depict data from 1203 adult patients with IBS-C in two randomized, double-blind, placebo-controlled clinical trials (trial 1 and trial 2). Patients were randomized and observed for period of 52 weeks, formulated to placebo or Tenapanor $50 \mathrm{mg}$ twice daily. Demographic profile of both the trial group were comparable. ${ }^{26}$

Double-blind, placebo-controlled, randomized, multicenter trials in adult patients was conducted to evaluate the clinical efficacy of Tenapanor for IBS-C: Trial 1 (TEN-01-302; NCT02686138) and Trial 2 (TEN01-301; NCT02621892). ${ }^{26}$ The population under study included 620 patients in trial 1 and 606 patients in trial 2 with mean age of 46 years (range 18 to 75 years), $80 \%$ females, $64 \%$ White and $31 \%$ Black/African American. ${ }^{26}$ Protocol under trial in these studies included, Tenapanor administration immediately prior to breakfast or the first meal of the day and immediately prior to dinner. ${ }^{26}$

\section{Inclusion criteria}

Rome III criteria for IBS-C for all patients. Clinical criteria during the 2 -week was abdominal pain score of at least 3 on a 0 -to-10-point numeric rating scale where a score of 0 indicates no pain and 10 indicates very severe pain, less than 3 complete spontaneous bowel movements (CSBMs) per week and less than or equal to 5 SBMs per week. ${ }^{26}$ Identical trial designs were followed in both trials till 12 weeks thereafter in trial one additional 14 week treatment ( 26 week double blind trial) and trial 2 included 4 week randomised withdrawal. ${ }^{26}$ Primary endpoint was the proportion of responders, where a responder was defined as a patient achieving both the stool frequency and abdominal pain intensity responder criteria in the same week for at least 6 of the first 12 weeks of treatment. ${ }^{26}$

The stool frequency (CSBM) and abdominal pain responder criteria for weekly assessment were formulated. ${ }^{26}$

CSBM responder: increase by least $1 \mathrm{CSBM}$ in weekly average from baseline.

Abdominal pain responder: $30 \%$ reduction in the weekly average score compared with baseline.

The responder rates for the primary endpoint and components of the primary endpoint (CSBM and abdominal pain), which were pre-specified key secondary end points, are shown in (Table 1). ${ }^{26}$

Table 1: Efficacy responder rates in placebo-controlled trials (trial 1 and trial 2) in adults with IBS-C: responder for at least 6 of the first 12 weeks of treatment.

\begin{tabular}{|c|c|c|c|}
\hline \multicolumn{3}{|l|}{ Responder rates } & \multirow[b]{2}{*}{ Treatment difference $\left(95 \% \mathrm{CI}^{\mathrm{a}}\right)$} \\
\hline Trial 1 & $\begin{array}{l}\text { Tenapanor } \\
(\%)(n=293)\end{array}$ & $\begin{array}{l}\text { Placebo }(\%) \\
(n=300)\end{array}$ & \\
\hline Responder $^{\mathrm{b}}$ components of responder endpoint & 37 & 24 & \multirow{3}{*}{$13 \%(6 \%, 20 \%)$} \\
\hline CSBM responder ${ }^{\mathrm{c}}$ & 47 & 33 & \\
\hline Abdominal pain responder ${ }^{\mathrm{d}}$ & 50 & 38 & \\
\hline Trial 2 & $\begin{array}{l}\text { Tenapanor } \\
(\%)(n=307)\end{array}$ & $\begin{array}{l}\text { Placebo }(\%) \\
(n=299)\end{array}$ & \\
\hline Responder $^{\mathrm{b}}$ components of responder endpoint & 27 & 19 & \multirow{3}{*}{$8 \%(2 \%, 15 \%)$} \\
\hline CSBM responder ${ }^{\mathrm{c}}$ & 34 & 29 & \\
\hline Abdominal pain responder ${ }^{\mathrm{d}}$ & 44 & 33 & \\
\hline
\end{tabular}

${ }^{a} \mathrm{CI}$ : Confidence Interval; ${ }^{\mathrm{b}} \mathrm{a}$ responder for these trials was defined as a patient who met both the abdominal pain and CSBM weekly responder criteria for at least 6 of the first 12 weeks; ${ }^{c}$ a CSBM responder was defined as a patient who achieved an increase in at least 1 CSBM per week, from baseline, for a least 6 of at least 12 weeks; ${ }^{d}$ an abdominal pain responder was defined as a patient who met the criteria of at least $30 \%$ reduction from baseline in weekly average of the worst daily abdominal pain, for at least 6 of the first 12 weeks.

In both trails the proportion of responders for 9 out of the first 12 weeks, including at least 3 of the last 4 weeks, was greater in Tenapanor treated patients compared to placebo-treated patients. ${ }^{26}$ There was considerable improvement noted in both trials by week with reference to CSBMs and the effect maintained till end of treatment. ${ }^{26}$
In trial 2 patients were re randomised and on average of 4 weeks observation CSBM and abdominal pain severity worsened. ${ }^{26}$ Patients continued on Tenapanor maintained their response to therapy and patients re randomised to Tenapanor had average increase in CSBM frequency and decreased abdominal pain. ${ }^{26}$ 


\section{APPROVAL}

Based on positive results from the phase III T3MPO trial program, Tenapanor has shown positive results for IBS-C management and received US FDA approval in September 2019 and soon will add to the existing modalities for management of IBS-C. ${ }^{26}$

\section{CONCLUSION}

With limited options for management of IBS-C, there was a definitive need for exploration of modalities for managing patients stressed due to residual symptoms, thereby hampering their social and mental productivity. Tenapanor, a novel molecule has shown promising results and would be of extreme morale boosting to the patients and clinician managing IBS-C with improved efficacy with least side effects and residual symptoms.

\section{Funding: No funding sources}

Conflict of interest: None declared

Ethical approval: Not required

\section{REFERENCES}

1. Longstreth GF, Thompson WG, Chey WD, Houghton LA, Mearin F, Spiller RC. Functional bowel disorders. Gastroenterology. 2006;130:1480-91.

2. Canavan C, West J, Card T. The epidemiology of irritable bowel syndrome. Clin Epidemiol. 2014;6:7180 .

3. Chey WD, Kurlander J, Eswaran S. Irritable bowel syndrome: a clinical review. JAMA. 2015;313:94958.

4. Drossman DA, Camilleri M, Mayer EA, Whitehead WE. AGA technical review on irritable bowel syndrome. Gastroenterology. 2002;123:2108-31.

5. El-Serag HB, Olden K, Bjorkman D. Health-related quality of life among persons with irritable bowel syndrome: a systematic review. Aliment Pharmacol Ter. 2002;16:1171-85.

6. Mayer EA. Clinical practice. Irritable bowel syndrome. N Engl J Med. 2008;358:1692-9.

7. Frank L, Kleinman L, Rentz A, Ciesla G, Kim JJ, Zacker C. Health-related quality of life associated with irritable bowel syndrome: comparison with other chronic diseases. Clin Ther. 2002;24:675-89.

8. Gralnek IM, Hays RD, Kilbourne A. The impact of irritable bowel syndrome on health-related quality of life. Gastroenterology. 2000;119:654-60.

9. Pace F, Molteni P, Bollani S, Sarzi-Puttini P, Stockbrügger R, Porro GB, et al. Inflammatory bowel disease versus irritable bowel syndrome: a hospitalbased, case-control study of disease impact on quality of life. Scand J Gastroenterol. 2003;38:1031-8.

10. Lacy BE, Chey WD, Lembo AJ. New and emerging treatment options for irritable bowel syndrome. Gastroenterol Hepatol (N Y). 2015;11:1-19.

11. Lembo A. Irritable bowel syndrome medications side effects survey. J Clin Gastroenterol. 2004;38:776-81.
12. Quigley EM, Tack J, Chey WD, Rao SS, Fortea J, Falques $\mathrm{M}$, et al. Randomised clinical trials: linaclotide phase 3 studies in IBS-C - a prespecified further analysis based on European Medicines Agency-specified endpoints. Aliment Pharmacol Ther. 2013;37:49-61.

13. Chey WD, Lembo AJ, Lavins BJ, Shiff SJ, Kurtz CB, Currie MG, et al. Linaclotide for irritable bowel syndrome with constipation: a 26-week, randomized, double-blind, placebo-controlled trial to evaluate efficacy and safety. Am J Gastroenterol. 2012; 107:1702-12.

14. Drossman DA, Chey WD, Johanson JF, Fass R, Scott C, Panas R, et al. Clinical trial: lubiprostone in patients with constipation-associated irritable bowel syndrome-results of two randomized, placebocontrolled studies. Aliment Pharmacol Ther. 2009;29:329-41.

15. Spencer AG, Labonte ED, Rosenbaum DP. Intestinal inhibition of the $\mathrm{Na}+\mathrm{H}+$ exchanger 3 prevents cardiorenal damage in rats and inhibits $\mathrm{Na}+$ uptake in humans. Sci Transl Med. 2014;6:227-36.

16. Johansson S, Rosenbaum DP, Knutsson M, Leonsson-Zachrisson M. A phase 1 study of the safety, tolerability, pharmacodynamics, and pharmacokinetics of tenapanor in healthy Japanese volunteers. Clin Exp Nephrol. 2017;21(3):407-16.

17. Eutamene H, Charmot D, Navre M, Bueno L. Visceral antinociceptive effects of RDX5791, a firstin-class minimally systemic NHE3 inhibitor on stress-induced colorectal hypersensitivity to distension in rats. Gastroenterology. 2011;140:S-578.

18. Zachos NC, Tse M, Donowotz M. Molecular Physiology of Instetine $\mathrm{Na} / \mathrm{H}$ +exchange. Annual review Physiol. 2005;67;411-43.

19. Charmot D. Non-systemic drugs: a critical review. Curr Pharm Des. 2012;18(10):1434-45.

20. Chey WD, Lembo AJ, Rosenbaum DP. Tenapanor Treatment of Patients With ConstipationPredominant Irritable Bowel Syndrome: A Phase 2, Randomized, Placebo-Controlled Efficacy and Safety Trial. Am J Gastroenterol. 2017;112(5):763-74.

21. Rosenbaum DP, Yan A, Jacobs JW. Pharmacodynamics, Safety, and Tolerability of the NHE3 Inhibitor Tenapanor: Two Trials in Healthy Volunteers. Clin Drug Investig. 2018;38(4):341-51.

22. Johansson S, Rosenbaum DP, Ahlqvist M, Rollison H, Knutsson M, Stefansson B, et al. Effects of Tenapanor on Cytochrome P450-Mediated DrugDrug Interactions. Clin Pharmacol Drug Dev. 2017;6(5):466-75.

23. Johansson S, Rosenbaum D, Ahlqvist M, Rollison H, Knutsson M, Stefánsson B. Effects of Tenapanor on Cytochrome P450-Mediated Drug-Drug Interactions. Clin Pharmacol Drug Dev. 2017;6(5):466-75.

24. Brandsch M. Drug transport via the intestinal peptide transporter PepT1. Current Opinion Pharmacol. 2013;13(6):881-7. 
25. Johansson S, Rosenbaum DP, Johan S, Bergur K, Mikael L, Eleanor H, et al. Tenapanor administration and the activity of the $\mathrm{H}+$-coupled transporter PepT1 in healthy volunteers: Tenapanor and PepT1mediated drug-drug interactions. $\mathrm{Br} \mathrm{J}$ Clin Pharmacol. 2017.

26. Chey WD, Lembo AJ, Rosenbaum DP. Efficacy of Tenapanor in Treating Patients With Irritable Bowel Syndrome With Constipation: A 12-Week, Placebo-
Controlled Phase 3 Trial (T3MPO-1). Am J Gastroenterol. 2020;115(2):281-93.

Cite this article as: Brashier DBS, Kumar A, Singh A, Pratap U. Tenapanor: new approach to counter irritable bowel syndrome with constipation. Int $\mathbf{J}$ Basic Clin Pharmacol 2020;9:1180-5. 